\title{
Propuesta de infraestructuras hidráulicas para el abastecimiento de la fauna de la Reserva Valenciana de Caza de la Muela de Cortes (RVCMC)
}

\author{
Ferrer Gisbert, A. ${ }^{1}$; García Post, R. ${ }^{2}$; Segarra Vidal, J. ${ }^{*}$ y Torregrosa Soler, J.B. ${ }^{1}$ \\ ${ }^{1}$ Universidad Politécnica de Valencia \\ ${ }^{2}$ Generalitat Valenciana \\ e-mail: *joasevi@etsiamn.upv.es
}

\section{Resumen}

La Reserva Valenciana de Caza de la Muela de Cortes, está situada en el centro occidental de la provincia de Valencia y tiene una superficie del orden de la 36,000 hectáreas, y presenta la problemática de que, aun existiendo, grandes masas de agua en sus lindes norte y oeste (río Júcar, con algunas presas), así como diversos puntos de agua en su interior, existe una amplia zona del orden de 6,000 hectáreas con serios problemas de abastecimiento que provoca que la fauna de dicha Reserva, o bien abandone la zona o tenga que cubrir grandes distancias para satisfacer sus necesidades, o se concentre en determinados puntos, lo que favorece, la depredación, competencia y transmisión de enfermedades. Es por ello que se proponen diversas alternativas para la solución del problema que se exponen en el presente artículo.

Palabras clave: fauna, recursos hídricos, Reserva Valenciana de Caza de la Muela de Cortes, diques, bebederos, aljibes, atrapanieblas 


\section{Antecedentes y problemática}

La Reserva Valenciana de Caza de la Muela de Cortes (en adelante RVCMC), está situada en el centro occidental de la provincia de Valencia y tiene una superficie del orden de la 36,000 hectáreas, y presenta la problemática de que, aun existiendo, grandes masas de agua en sus lindes norte y oeste (río Júcar, con algunas presas), así como diversos puntos de agua en su interior, existe una amplia zona del orden de 6,000 hectáreas con serios problemas de abastecimiento que provoca que la fauna de dicha Reserva, o bien abandone la zona o tenga que cubrir grandes distancias para satisfacer sus necesidades, o se concentre en determinados puntos, lo que favorece, la competencia y transmisión de enfermedades entre otras cosas.

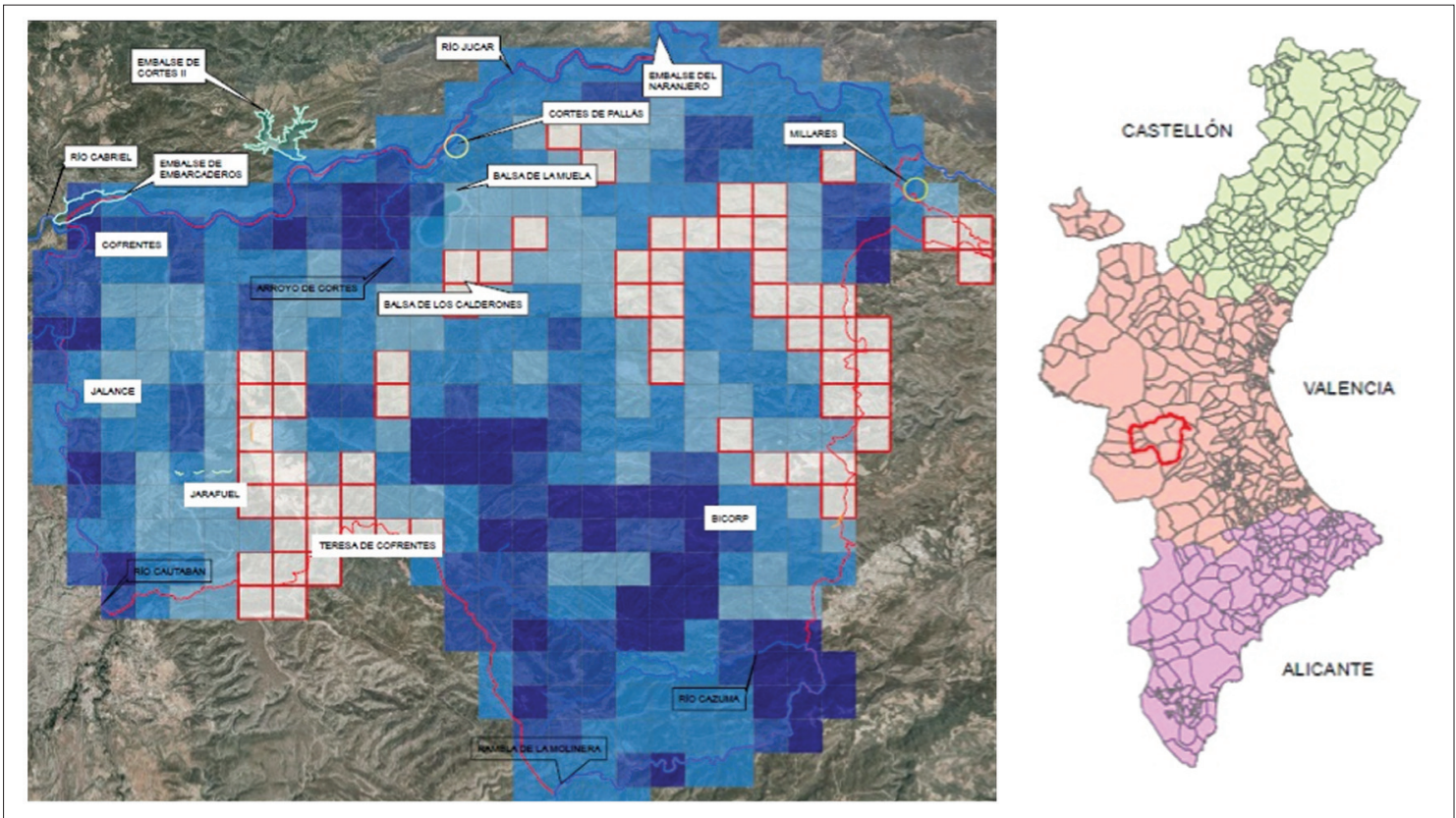

Figura 1. Situación de la Reserva, escala 1:100.000 (Lluna, 2012).

Las teselas de color blanco, son las más deficitarias y son sobre las que se planifica la actuación.

\section{Objeto}

El objeto del presente trabajo consiste en analizar y establecer una serie de propuestas técnicas que individualmente o interconectadas resuelvan la escasez de recursos hídricos de la fauna de la reserva.

\section{Necesidades a cubrir}

Las necesidades a cubrir se establecen en función de la especies y del número de cada una de ellas de tal forma que se garanticen sus necesidades hídricas. 
Las especies cinegéticas, su población actual y futuro se resumen en la Tabla 1 (VAERSA-Generalitat Valenciana, 2012).

Tabla 1. Especies cinegéticas.

\begin{tabular}{lccc}
\hline \multicolumn{1}{c}{ Nombre científico } & Nombre en castellano & Contingentes actuales & Contingentes potenciales \\
\hline Alectoris rufa & Perdiz & 688 & 4,199 \\
\hline Oryctolagus cuniculus & Conejo & 1,341 & 6,029 \\
\hline Lepus granatensis & Liebre & 180 & 532 \\
\hline Columba palumbus & Paloma torcaz & 723 & - \\
\hline Streptopelia turtur & Tórtola común & 420 & - \\
\hline Turdus sp. & Zorzal & 886 & - \\
\hline Capra pirenaica & Cabra montés & 1,835 & 1,639 \\
\hline Ovis musimon & Muflón & 2,800 & 1,199 \\
\hline Cervus elaphus & Ciervo & 245 & 90 \\
\hline Dama dama & Gamo & 110 & 75 \\
\hline Sus scrofa & Jabalí & 560 & 1,271 \\
\hline
\end{tabular}

Para la obtención de los consumos individuales de los animales se han consultado diversas fuentes, ante la escasez de datos de consumo de especies cinegéticas se ha optado por tomar los datos de animales tabulados. Por lo que se han seguido entre otras las indicadas de Anaya Garduño M. (2011), estableciéndose los siguientes consumos en la Tabla 2 que deben entenderse como consumos de cálculo para las zonas deficitarias.

Tabla 2. Consumos de cálculo para las teselas deficitarias.

\begin{tabular}{|c|c|c|c|c|}
\hline & $\mathrm{N}^{\mathrm{o}}$ de animales & $\begin{array}{l}\text { Consumo individual } \\
\text { (litros/día) }\end{array}$ & $\begin{array}{c}\text { Consumo } \\
\text { Total anual }\left(\mathrm{m}^{3}\right)\end{array}$ & $\begin{array}{c}\text { Consumo solo } \\
\text { verano }\left(\mathrm{m}^{3}\right)\end{array}$ \\
\hline $\begin{array}{l}\mathrm{N}^{o} \text { animales } \\
\text { caza mayor }\end{array}$ & 5,500 & 3 & 6,000 & \\
\hline $\begin{array}{l}\mathrm{N}^{\mathrm{o}} \text { animales } \\
\text { caza menor }\end{array}$ & 4,000 & 1 & 1,500 & \\
\hline Total & & & se aproxima a 8,000 & 2,000 \\
\hline
\end{tabular}

\section{Alternativas analizadas}

Las alternativas analizadas son:

1. Utilización de tractor con remolque cuba.

2. Construcción de charcas para el almacenamiento de escorrentías.

3. Aprovechamiento de las escorrentías de cubiertas de edificios aislados del interior de la reserva.

4. Ejecución de microcuencas impermeabilizadas artificialmente.

5. Captadores de niebla.

6. Otras alternativas. 


\subsection{Utilización de tractor con remolque cuba}

La utilización de un vehículo para el transporte de agua es la única forma de la que se dispone en la actualidad para tratar de paliar la escasez de recursos hídricos que se dan en las zonas que según Lluna (2012) se corresponden con cuadrículas de prioridad máxima, debido a que estas zonas no disponen de ninguna infraestructura, fuente, etc. que permita a la fauna acceder al agua.

El método que se utiliza actualmente para el suministro de agua para las mencionadas zonas consiste en el transporte de depósitos de polietileno de $1 \mathrm{~m}^{3}$ de capacidad.

De los distintos tipos de vehículos analizados, el que se ha mostrado más eficaz es un tractor agrícola con tracción a las cuatro ruedas y un remolque cuba de 10-15 $\mathrm{m}^{3}$ de capacidad. Esta solución supone que una cuba de $10 \mathrm{~m}^{3}$, con un rendimiento de 300 días al año y un viaje al día, podría aportar $3.000 \mathrm{~m}^{3}$ anuales para el abastecimiento de la fauna en las zonas que existe una escasez de recursos hídricos.

\subsection{Construcción de charcas para almacenamiento de escorrentías}

Fijada una localización se obtiene:

- Capacidad mínima necesaria del vaso.

- Aporte de la cuenca que alimenta el vaso.

- Caudal máximo de escorrentía para un periodo de retorno de 100 años.

Se analiza además el cierre del vaso natural con un dique de tierra y con un dique de hormigón, en ambos casos se impermeabiliza el vaso con geomembranas.

Para la determinación del complejo hidrológico "suelo-cubierta" anual medio se debe recordar que la cuenca elegida tiene las siguientes características:

- Uso del suelo: Matorral-Pastizal mediterráneo $\mathrm{FCC}=50 \%$.

- Litología: Calcáreas.

- Textura: Franco-arcillosa.

- Fisiografía: Fuertemente socavado.

- Superficie: 87,11 ha.

De acuerdo con el Instituto Nacional de Reforma y Desarrollo Agrario-Ministerio de Agricultura, Pesca y Alimentación (1985) en NC sería 86, ya que a pesar de tener una cobertura media las condiciones hidrológicas para la infiltración son muy pobre debido a la poca profundidad y el poco grado de consolidación de las capas de mantillo y humus del monte bajo. Además, se sitúa en el Grupo 3 de los grupos hidrológicos debido a que la textura del lugar es franco-arcillosa.

El volumen originado por las escorrentías de la cuenca que abastece al vaso se ha obtenido siguiendo el método conocido como Número de Curva o Método Analítico (Unites States Departament of Agriculture (USDA), 2004), siguiendo las recomendaciones del manual "Diseño y construcción de pequeños embalses" (Instituto Nacional de Reforma y Desarrollo Agrario-Ministerio de Agricultura, Pesca y Alimentación, 1985), tras lo cual se obtiene la Tabla 3. 
Tabla 3. Volumen generado por las escorrentías de la cuenca para un año medio.

\begin{tabular}{l|c|c|c|c|c|c|c|c|c|c|c|c|c}
\hline Mes & May. & Jun. & Jul. & Agost. & Sept. & Oct. & Nov. & Dic. & Enero & Febr. & Marz. & Abril & Total \\
\hline $\mathrm{V}\left(\mathrm{m}^{3}\right)$ & 55.64 & 0.00 & 0.00 & 0.00 & 182.98 & $12,643.45$ & $5,266.44$ & $3,134.40$ & $2,452.21$ & $2,799.40$ & $2,965.37$ & $2,772.22$ & $32,272.12$ \\
\hline
\end{tabular}

El caudal máximo, a evacuar por el aliviadero que se diseña posteriormente, se ha calculado siguiendo la metodología propuesta de Témez Peláez (1978) para pequeñas cuencas utilizando la ley de distribución de Gumbel (1967). Se adjuntan en la Tabla 4 los valores obtenidos para distintos periodos de retorno.

Tabla 4. Caudal máximo de escorrentía de la cuenca para distintos periodos de retorno.

\begin{tabular}{l|c|c|c|c|c|c|c|c|c|c|c}
\hline Período retorno-T (años) & 2 & 5 & 10 & 25 & 50 & 75 & 100 & 250 & 500 & 1,000 & 10,000 \\
\hline $\mathrm{Q}\left(\mathrm{m}^{3} / \mathrm{s}\right)$ Caudal de máxima avenida & 4.40 & 5.83 & 6.83 & 8.17 & 9.20 & 9.81 & 10.25 & 11.68 & 12.78 & 13.91 & 17.77 \\
\hline
\end{tabular}

Para el cierre de dique se han analizado dos tipologías de presa, de tierra y fabrica, que se comentan a continuación:

\subsubsection{Charca con dique de tierra}

Una vez fijada la localización se puede empezar a realizar las primeras mediciones con tal de asegurar que la charca tenga la suficiente capacidad como para almacenar un volumen aproximado de agua de 9,000 m3. Con esta capacidad se garantiza que para un llenado que se produzca en otoño, se tenga un volumen suficiente disponible para la fauna hasta el otoño siguiente, en el supuesto de que no se produjese ninguna aportación al vaso el resto del año, según se observa en la Figura 2.

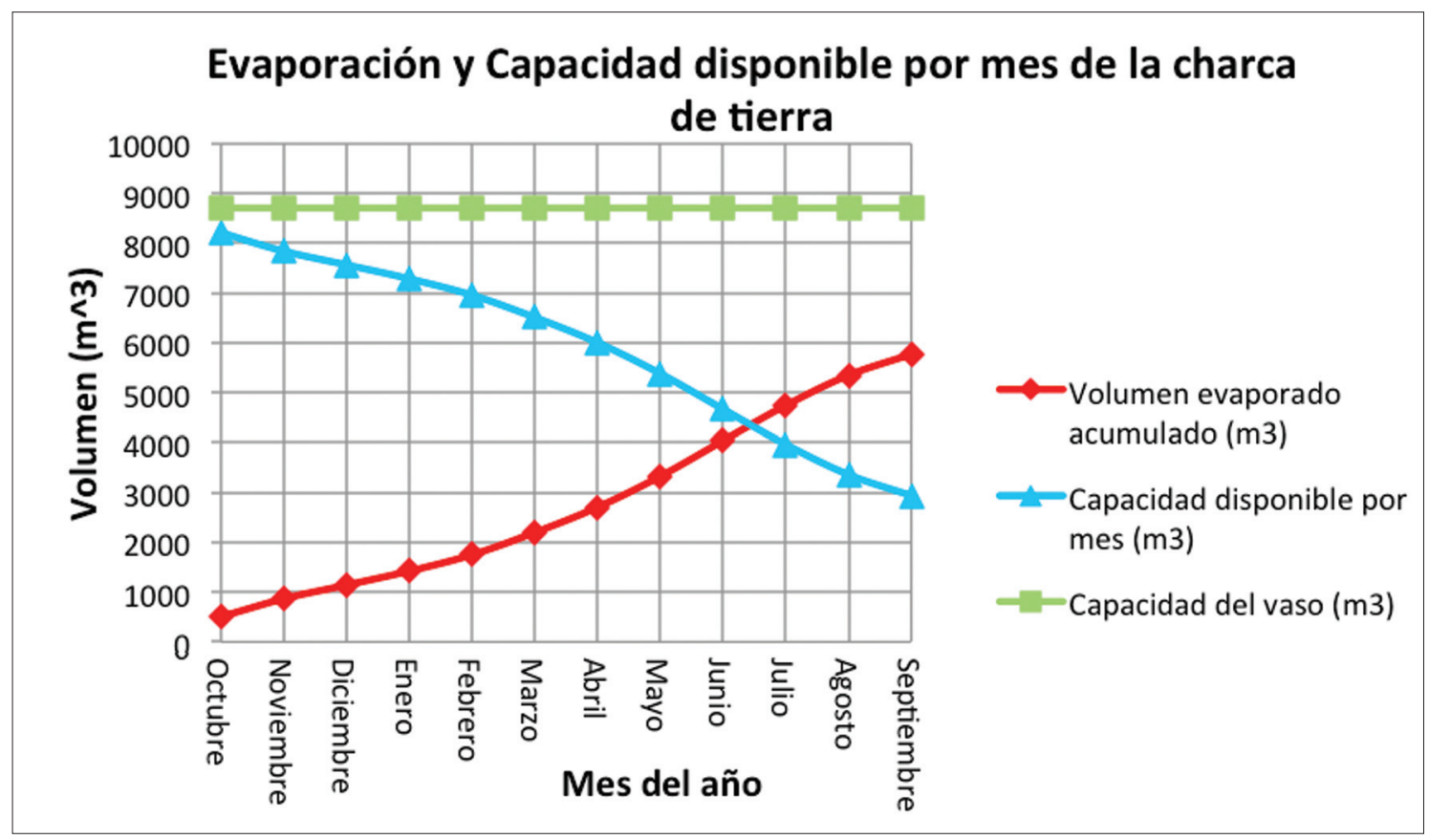

Figura 2. Capacidad disponible para la fauna tras un llenado en otoño. 
Para este caso las características de la sección son las que se muestran en la Tabla 5.

Tabla 5. Características principales de la sección del dique de tierra

\begin{tabular}{ll}
\hline Talud aguas arriba & $5 \mathrm{H} / 1 \mathrm{~V}$ \\
\hline Talud aguas abajo & $2 \mathrm{H} / 1 \mathrm{~V}$ \\
\hline Altura hasta cimiento & 9 metros \\
\hline Anchura de coronación & 5 metros \\
\hline Dren estructural & Tipo chimenea \\
\hline Aliviadero & $10.25 \mathrm{~m}^{3} \mathrm{~s}^{-1}$ sobre el talud exterior protegido \\
\hline
\end{tabular}

Todo el vaso, incluyendo el talud interior se encuentra impermeabilizado con geosintéticos suponiendo unas filtraciones nulas. La secuencia de materiales de abajo hacia arriba es la siguiente:

1. Capa de asiento del terreno refinada y nivelada, exenta de material grueso.

2. $15 \mathrm{~cm}$ de arena.

3. Geotextil de polipropileno con una densidad de $400 \mathrm{~g} \mathrm{~m}^{-2}$.

4. Geomembrana de Policloruro de Vinilo de $1.5 \mathrm{~mm}$ de espesor.

5. Geotextil de polipropileno con una densidad de $400 \mathrm{gr} \mathrm{m}^{-2}$.

6. Capa de gravilla 10/12 de $15 \mathrm{~cm}$ de espesor.

7. Capa de grava 50/60 de $20 \mathrm{~cm}$ de espesor.

8. Rip-rap.

A continuación se muestra un croquis de la sección tipo en la Figura 3.

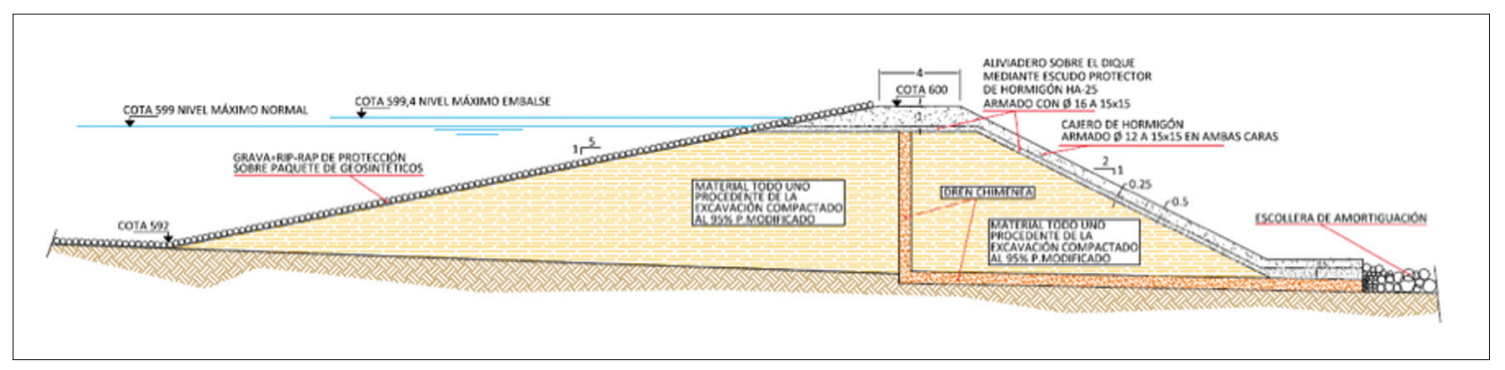

Figura 3. Croquis sección tipo.

\subsubsection{Charca con dique de hormigón}

En el caso del dique de fabrica la solución empleada en el vaso es idéntica a la empleada con el dique de tierra, la diferencia básica estriba en una mayor capacidad $10,779.5 \mathrm{~m}^{3}$ a la misma cota de máximo embalse normal, y por lo tanto de volumen disponible útil según de observa en la Figura 4.

Siguiendo la metodología de Vallarino Cánovas del Castillo (1991) se ha realizado para distintos valores del ángulo de rozamiento, el peso específico de la fábrica, 


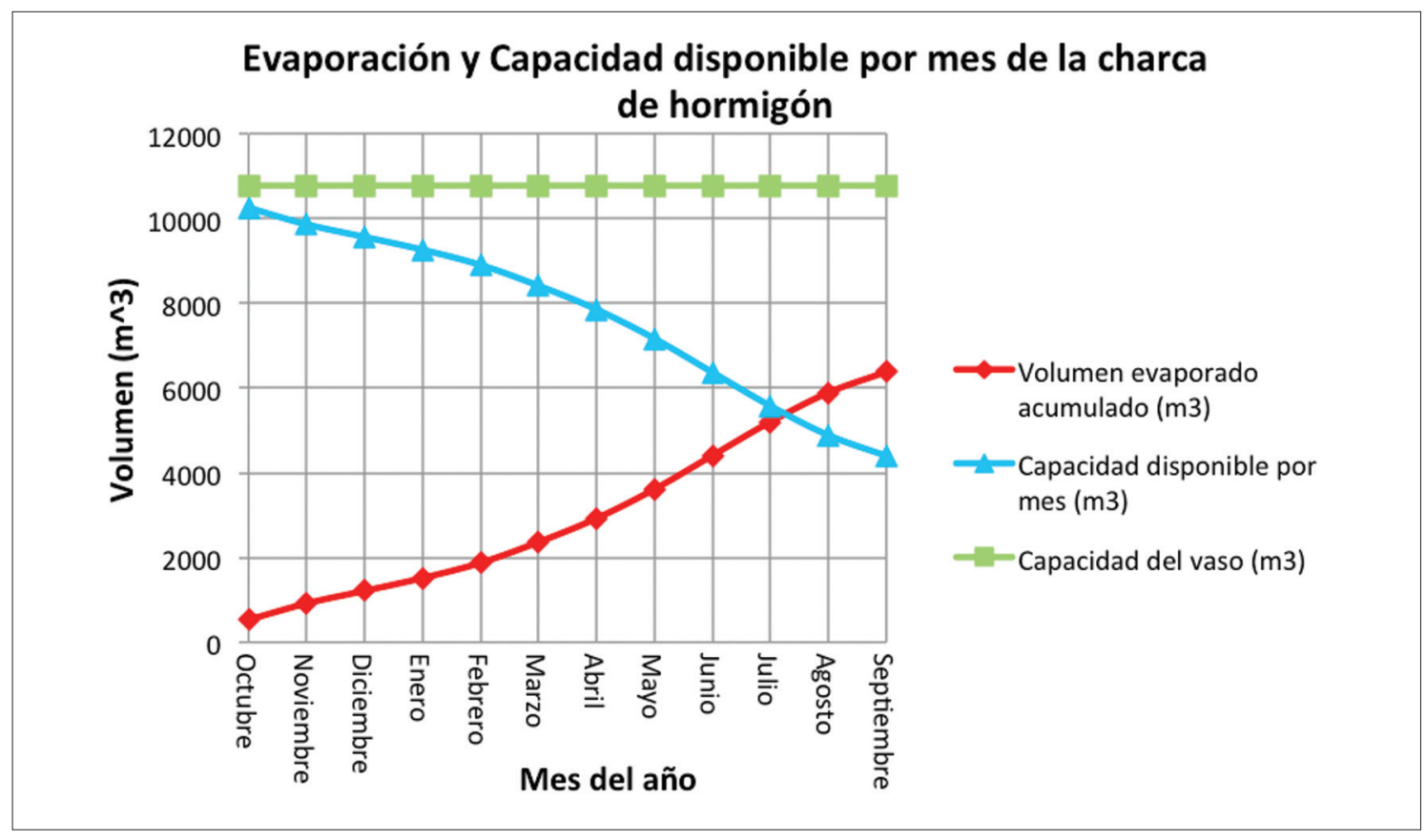

Figura 4. Evaporación y capacidad disponible por mes de la charca de hormigón.

y coeficiente de seguridad al deslizamiento "K.", la relación volumen unitario frente al coeficiente " $\alpha$ " de la subpresión. A continuación se presentan las gráficas obtenidas con los siguientes valores: $\varphi=35^{\circ}, \gamma_{\mathrm{c}}=2,400 \mathrm{Kg} \mathrm{m}^{\wedge-3}, \mathrm{~K}_{1}=1.35$

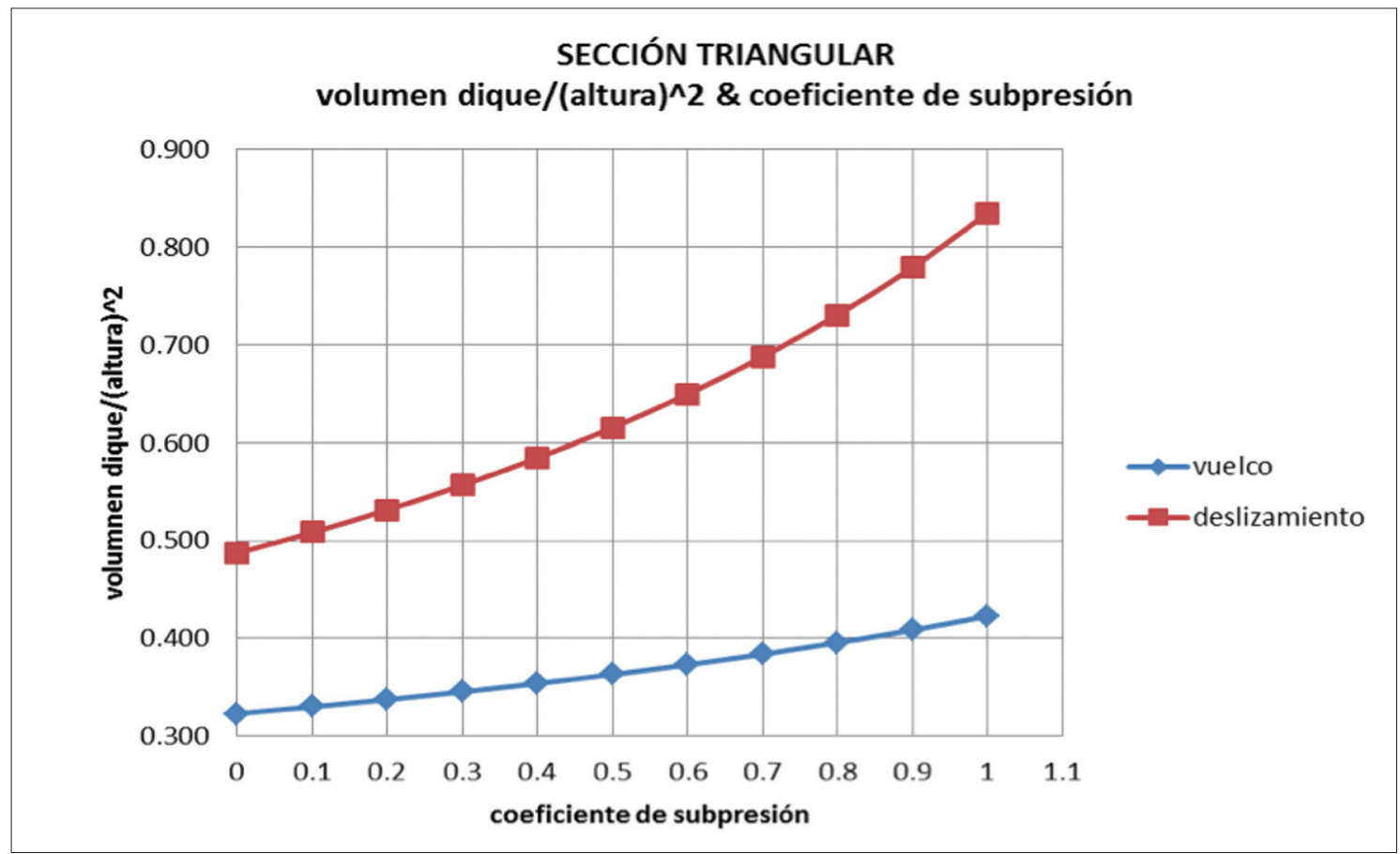

Figura 5. Relación volumen unitario/altura al cuadrado frente al coeficiente de subpresión para una sección transversal triangular, por vuelco y deslizamiento. 


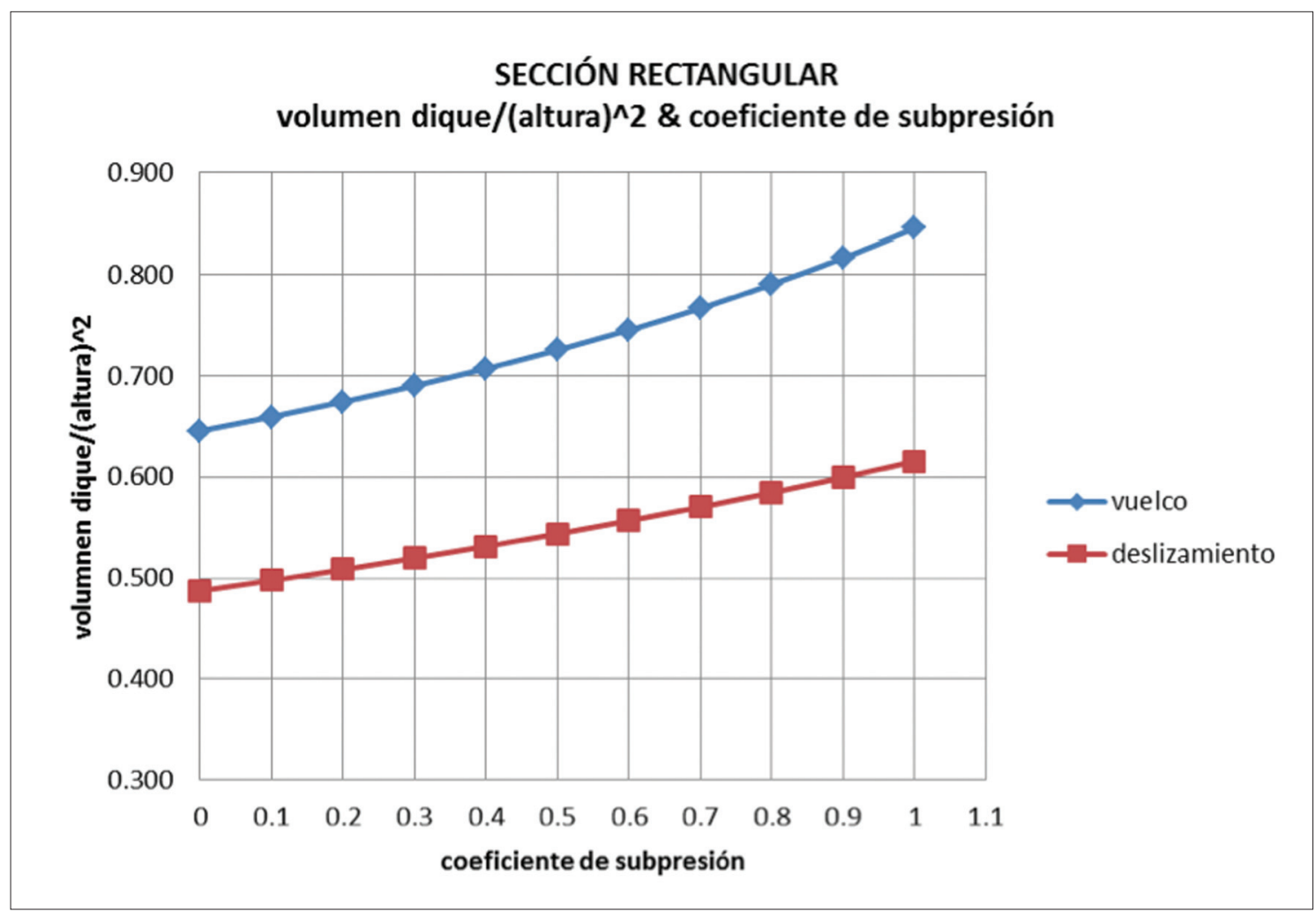

Figura 6. Relación volumen unitario/altura al cuadrado frente al coeficiente de subpresión para una sección transversal rectangular, por vuelco y deslizamiento.

La tipología de la sección elegida es la triangular, ya que es la que necesita menos material, y en principio más económica que la rectangular, para las mismas condiciones de seguridad.

Para la sección triangular los parámetros de cálculo utilizados son:

1. A efectos de vuelco, se impone la condición de que la tensión transmitida al terreno aguas arriba, sea nula.

2. No se considera la cohesión para la comprobación de deslizamiento.

3. Se utiliza un coeficiente frente al deslizamiento, considerando solo el rozamiento " $\mathrm{K}_{1}=1.35$ "

4. Se estima el ángulo de rozamiento del suelo en un valor de $35^{\circ}$

5. La tensión transmitida al terreno debe ser inferior a la admisible, que se considera de $0.245 \mathrm{MPa}$.

6. Se considera como coeficiente " $\alpha$ " de subpresión igual a la unidad.

7. Se considera que el material del dique es hormigón, con un peso específico de $2,400 \mathrm{~kg} \mathrm{~m}^{-3}$.

Para la pendiente del talud, se ha confeccionado la Figura 7.

Según la Figura 7, y para un coeficiente de subpresión la unidad, y un ángulo de rozamiento de $35^{\circ}$, la pendiente del talud es de 1.37, adoptándose el valor de 1.4.

Con las expresiones anteriormente expuestas, y una altura máxima de 9 metros, obtenemos: 


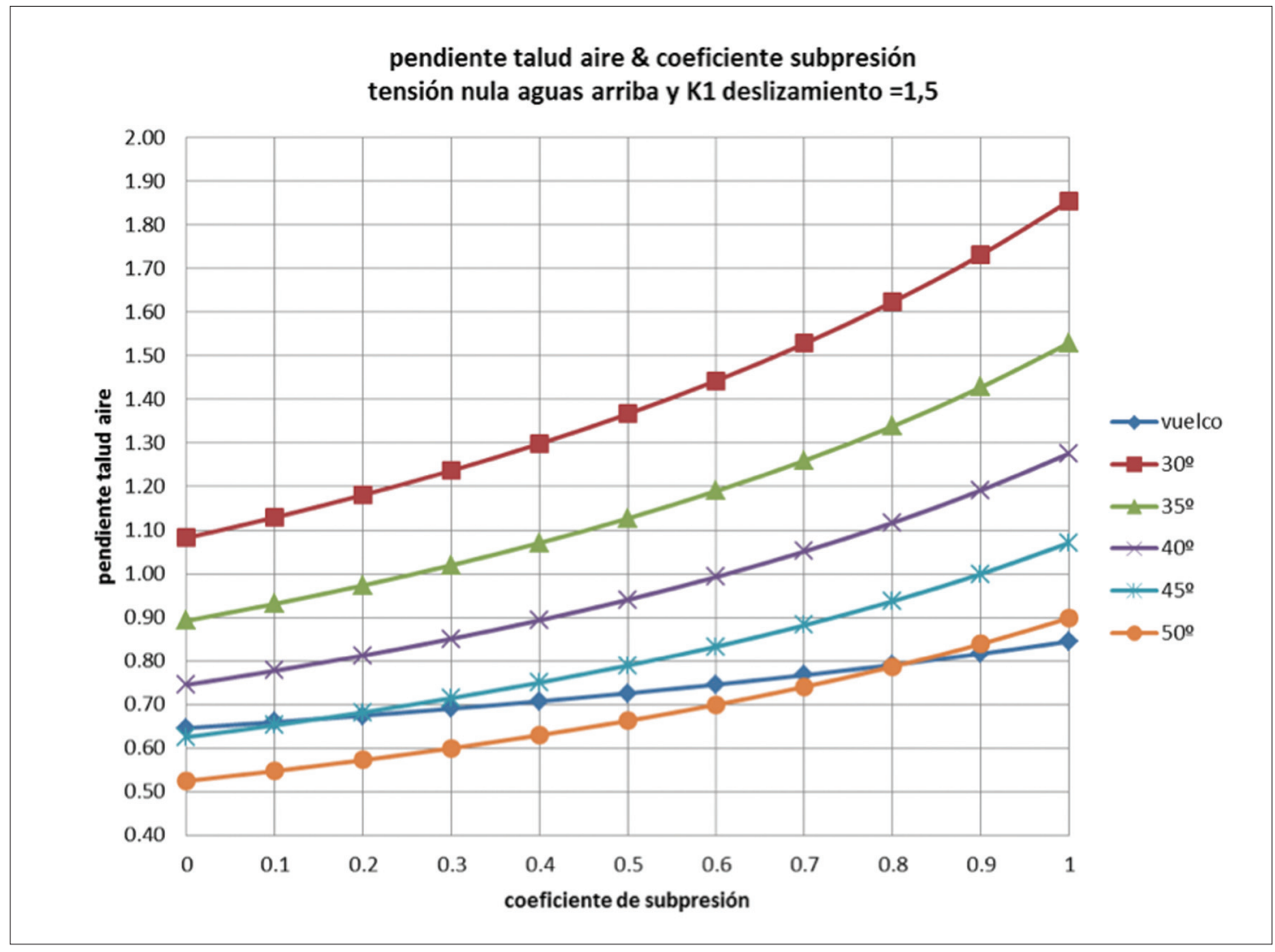

Figura 7. Obtención de la pendiente del talud en función de la subpresión por vuelco y deslizamiento.

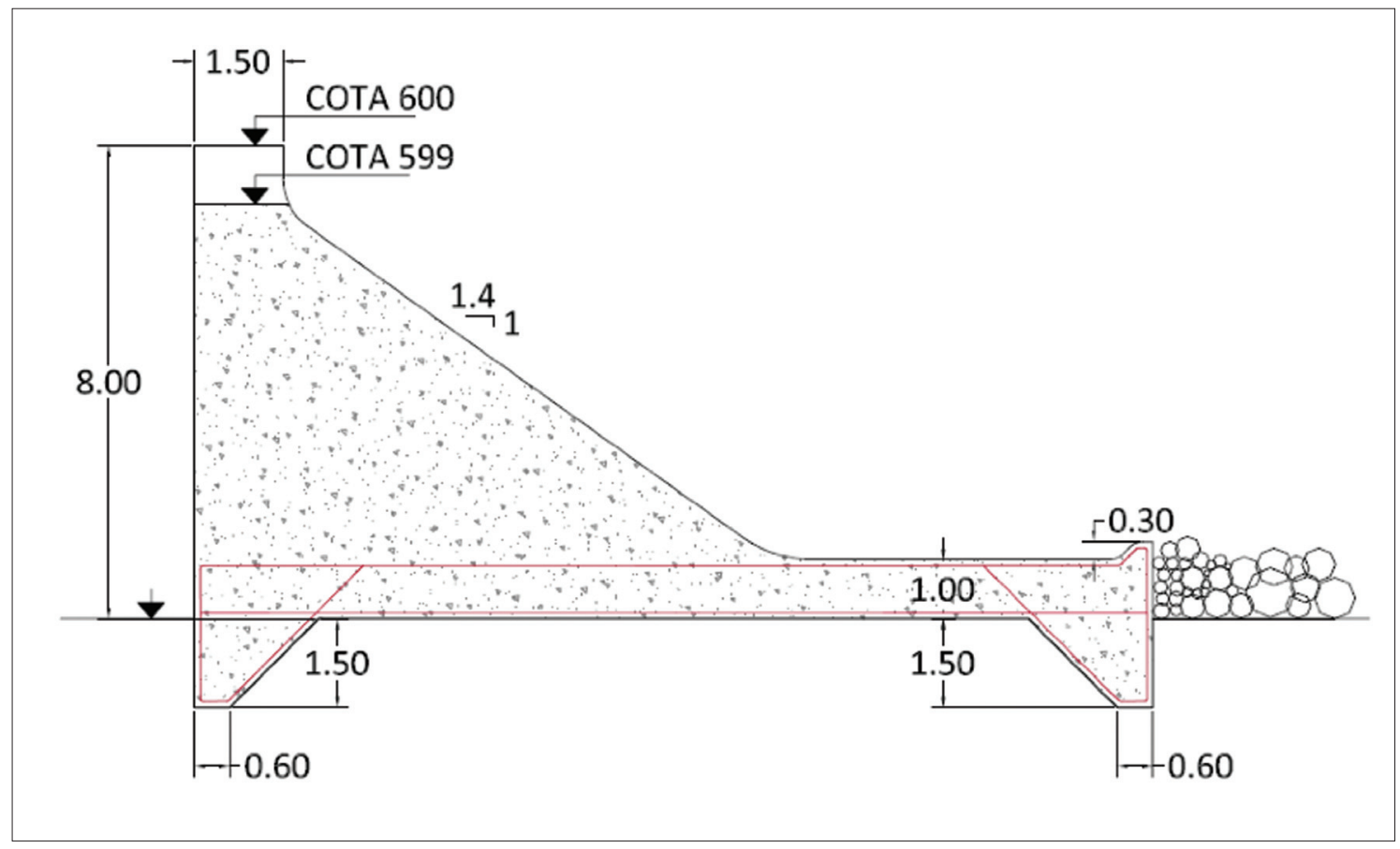

Figura 8. Esquema sección tipo dique hormigón. 
a). Hipótesis de carga con nivel de la lámina de agua en coronación:

- Tensión transmitida al terreno aguas arriba $=0.079 \mathrm{MPa}$.

- Tensión transmitida al terreno aguas abajo $=0.045 \mathrm{MPa}$.

Valores inferiores a la tensión admisible del terreno, estimada en 0.245 MPa.

b). Hipótesis de carga a peso propio exclusivamente:

- Tensión transmitida al terreno aguas arriba $=0.212 \mathrm{MPa}$.

- Tensión transmitida al terreno aguas abajo $=0 \mathrm{MPa}$.

Valores inferiores a la tensión admisible del terreno, estimada en 0.245 MPa.

Se presenta en la Figura 8 el esquema de la sección tipo.

\subsection{Aprovechamiento de las escorrentías de las cubiertas de edificios aislados del interior de la Reserva.}

En esta alternativa se analiza la posibilidad de aprovechar las casas rurales existentes como elemento de captación de lluvias, es decir actuando como un aljibe, almacenándolas en depósitos que posteriormente alimentaran los abrevaderos que estén directamente conectados a cada depósito.

Tabla 6. Número de casas de aperos con superficies mayores de $100 \mathrm{~m}^{2}$, su potencial de captación para el año medio y el tamaño del depósito recomendado.

\begin{tabular}{|c|c|c|c|c|c|}
\hline $\begin{array}{c}\text { Superficie } \\
\left(\mathrm{m}^{2}\right)\end{array}$ & $\begin{array}{c}\text { Números } \\
\text { de casas } \\
\text { de aperos }\end{array}$ & $\begin{array}{c}\text { Precipitación } \\
\text { efectiva captada } \\
\text { anual }\left(\mathrm{m}^{3}\right)\end{array}$ & $\begin{array}{c}\text { Precipitación } \\
\text { efectiva mensual } \\
\text { máxima }\left(\mathrm{m}^{3}\right)\end{array}$ & $\begin{array}{c}\text { Precipitación } \\
\text { efetiva mensual } \\
\text { del mes siguiente } \\
\text { al máximo }\left(\mathrm{m}^{3}\right)\end{array}$ & $\begin{array}{c}\text { Capacidad } \\
\text { depósito } \\
\text { recomendada }\left(\mathrm{m}^{3}\right)\end{array}$ \\
\hline $100-200$ & 82 & 49.6 & 8 & 5.3 & 20 \\
\hline $200-300$ & 24 & 82.6 & 13.4 & 8.8 & 30 \\
\hline $300-400$ & 14 & 115.7 & 18.7 & 12.3 & 40 \\
\hline$>400$ & 8 & 132.2 & 21.4 & 14 & 50 \\
\hline
\end{tabular}

Las capacidades recomendadas para cada depósito según la superficie de captación se resumen en la Tabla 7.

Las zonas de aplicación de este tipo de solución dentro de la reserva se ilustra en la Figura 9.

Se ha seleccionado un número de 16 casas de aperos de entre $100 \mathrm{~m}^{2}$ a $200 \mathrm{~m}^{2}$ ya que se ha realizado un análisis cartográfico con el objetivo de que las casas de aperos no se encuentren a poca distancia para evitar problemas de depredación, transmisión de enfermedades y competencia, por ello se ha fijado una distancia mínima de $500 \mathrm{~m}$ entre las 30 casas potencialmente utilizables en la zona sudoccidental de la RVCMC.

En la Tabla 7, se presenta el potencial de captación de recursos para el año medio en la zona suroccidental de la RVCMC. 


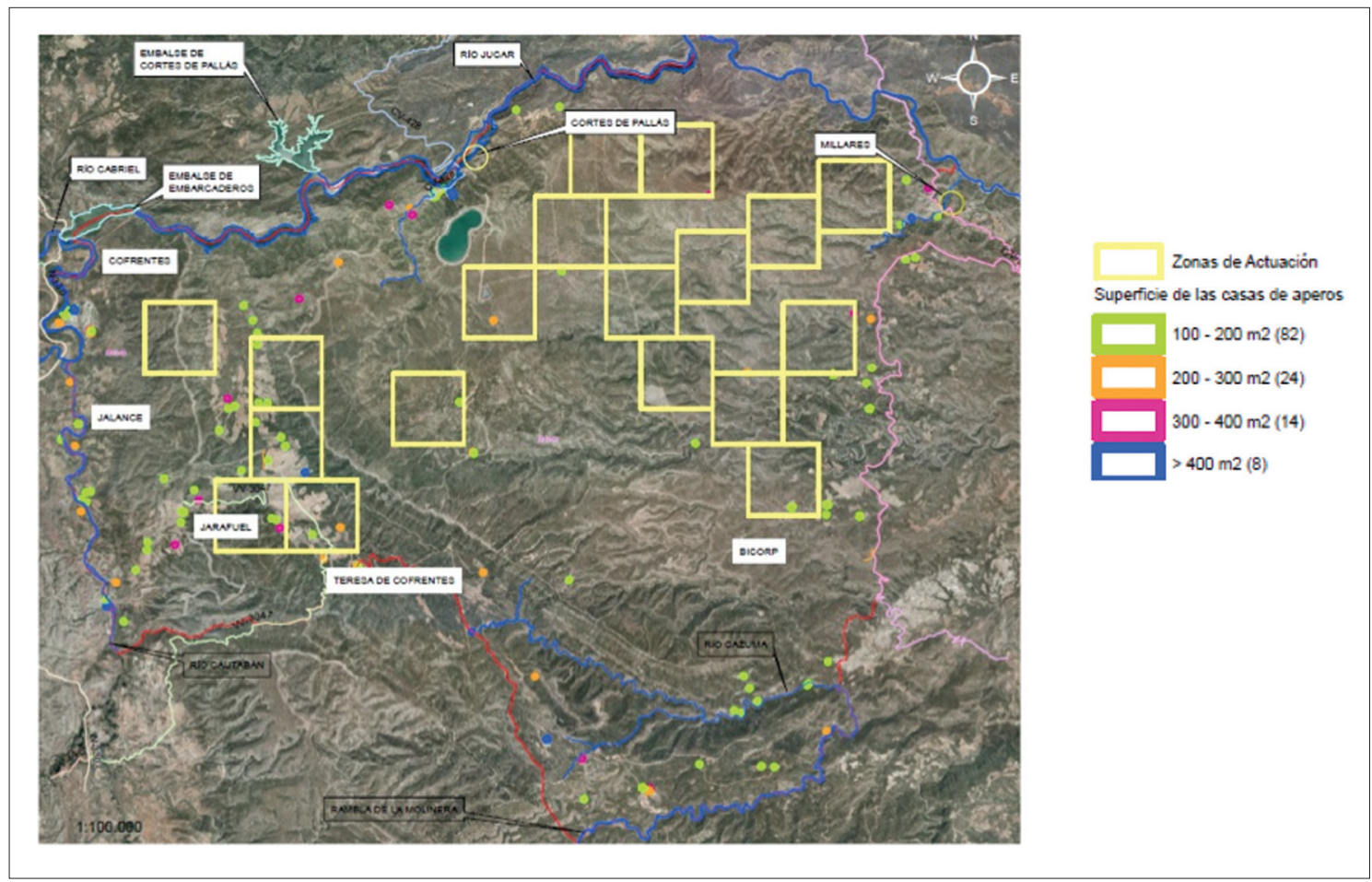

Figura 9. Imagen en la cual se puede observar unos cuadrados amarillos que representan las zonas donde se recomienda actuar en el estudio previo (Lluna, 2012), y también se observa la distribución de las casas de aperos que están representadas mediante puntos de diferentes colores según se indica en la leyenda de la parte superior derecha. Cada tesela es de $2 \mathrm{Km} \times 2 \mathrm{Km}$.

Tabla 7. Potencial de captación de aguas de las casas rurales para el año medio.

\begin{tabular}{|c|c|c|c|c|c|}
\hline $\begin{array}{c}\text { Superficie } \\
\left(\mathrm{m}^{2}\right)\end{array}$ & $\begin{array}{c}\text { Números } \\
\text { de casas } \\
\text { de aperos a } \\
\text { rehabilitar de } \\
\text { las existentes }\end{array}$ & $\begin{array}{c}\text { Precipitación } \\
\text { efectiva } \\
\text { captada } \\
\text { al año }\left(\mathrm{m}^{3}\right)\end{array}$ & $\begin{array}{c}\text { Precipitación } \\
\text { efectiva en los } \\
\text { meses previos } \\
\text { al verano } \\
\left.\text { (marzo-mayo } \mathrm{m}^{3}\right)\end{array}$ & $\begin{array}{c}\text { Precipitación } \\
\text { efetiva en los } \\
\text { meses de verano } \\
\left.\text { (junio-agosto } \mathrm{m}^{3}\right)\end{array}$ & $\begin{array}{c}\text { Captación } \\
\text { total estival }\left(\mathrm{m}^{3}\right)\end{array}$ \\
\hline $100-200$ & 16 & 49.6 & 14 & 4.9 & 78.4 \\
\hline $200-300$ & 2 & 82.6 & 23.4 & 8.2 & 16.4 \\
\hline $300-400$ & 2 & 115.7 & 32.8 & 11.5 & 23 \\
\hline$>400$ & 1 & 132.2 & 37.5 & 13.1 & 13.1 \\
\hline TOTAL & & & & & 130.9 \\
\hline
\end{tabular}

La cantidad almacenada de $131 \mathrm{~m}^{3}$ frente a $\operatorname{los} 2000 \mathrm{~m}^{3}$ necesarios, da una idea de lo deficitaria de esta solución para la resolución global de la escasez, aunque puede ser una alternativa puntual en algún caso.

\subsection{Ejecución de microcuencas impermeabilizadas artificialmente}

Ante la dificultad en cuanto a la disponibilidad de cubierta de casas rurales se 
puede sustituir la misma por una superficie de terreno, impermeabilizada con una geomembrana.

En la Fig. 10, se presenta la superficie necesaria en función de la capacidad que se desee disponer para un año de pluviometría medio.

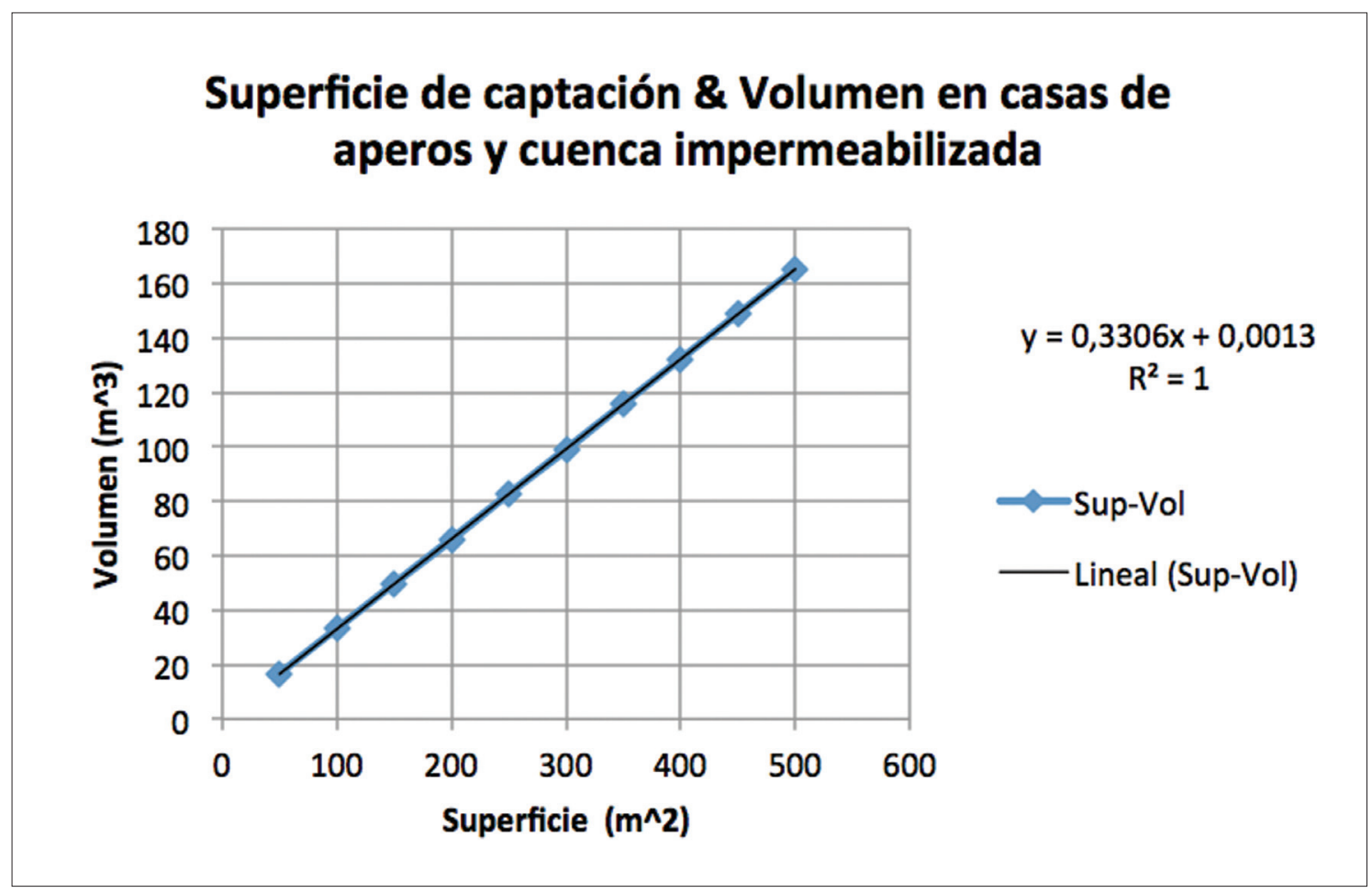

Figura 10. Relación superficie de captación frente a volumen captado en las alternativas de casas de aperos y cuenca impermeabilizadas.

\subsection{Captadores de niebla}

La utilización de captadores de niebla no resulta novedosa en el actual panorama de investigación sobre nuevos métodos de obtención de agua. Ya se viene evidenciando que los nuevos métodos de captación de precipitación horizontal presentan un potencial interés efectivo como se demuestra en el estudio de Corell et al (2011), en el cual se indica que un captador cilíndrico de pequeño tamaño $(0.5$ metros de alto por 0.25 de ancho aproximadamente) ha logrado captar alrededor de unos $211 \mathrm{~m}^{-2}$ día $^{-1}$ instalado en el Puig Neulós en Girona.

Se instaló un captador de niebla de similares características en una zona cercana al Cinto de la Cabra y otro captador en la zona de Bujete con el objetivo de evaluar la potencialidad de los lugares para la captación de precipitación horizontal. Del estudio realizado se extrajo la conclusión de que en la zona cercana al Cinto de la Cabra tenía mayor capacidad para captar agua proveniente de precipitación horizontal. 
Asimismo se instaló por parte de la entidad responsable de la RVCMC otro método de captación de agua de niebla de fabricación propia denominado como "árbol captador de niebla", este método se ha realizado intentando asemejar el captador de niebla a la forma de un árbol, en concreto de un pino. Como se puede observar en la Figura 11, el captador tiene una altura aproximada de $2 \mathrm{~m}$ y está formado por 15 brazos de PVC que se han ido acoplando a un eje central del mismo material mediante una ligadura de silicona.

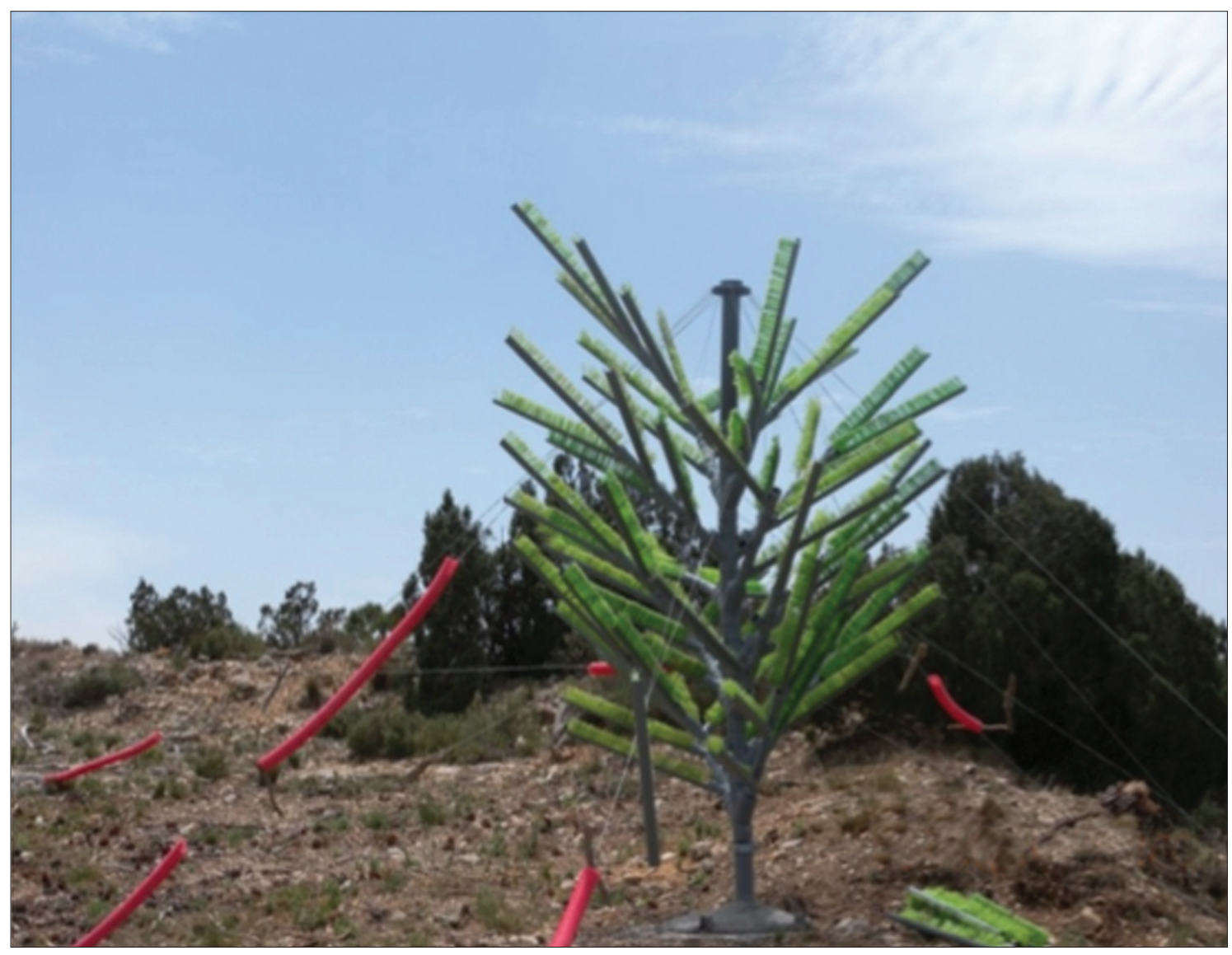

Figura 11. Imagen del método experimental de captación de agua proveniente de precipitación horizontal.

Cada uno de los brazos está relleno de unas "acículas" de plástico provenientes de una escoba convencional que simulan a efectos de captación las de un pino, para mayor detalle se debe decir que en cada brazo hay 5 ramas, en cada rama hay 70 ramilletes y en cada ramillete hay 24 "acículas" de plástico, con lo que si se asemeja la forma de una acícula de plástico a un cilindro y se supone que cada acícula de plástico tiene un diámetro de $0.8 \mathrm{~mm}$ y una altura de $10 \mathrm{~cm}$, supone que la superficie potencial de captación estimada es de $31.66 \mathrm{~m}^{2}$ por árbol.

Según los datos del estudio realizado por Corell et al (2011) en 8 meses, que van de enero a septiembre, se captaron $1,004.31 \mathrm{~m}^{-2}$ durante 91 días de niebla por lo que el potencial de captación en nuestro caso es de $31.8 \mathrm{~m}^{3}$ de agua captada por árbol en 


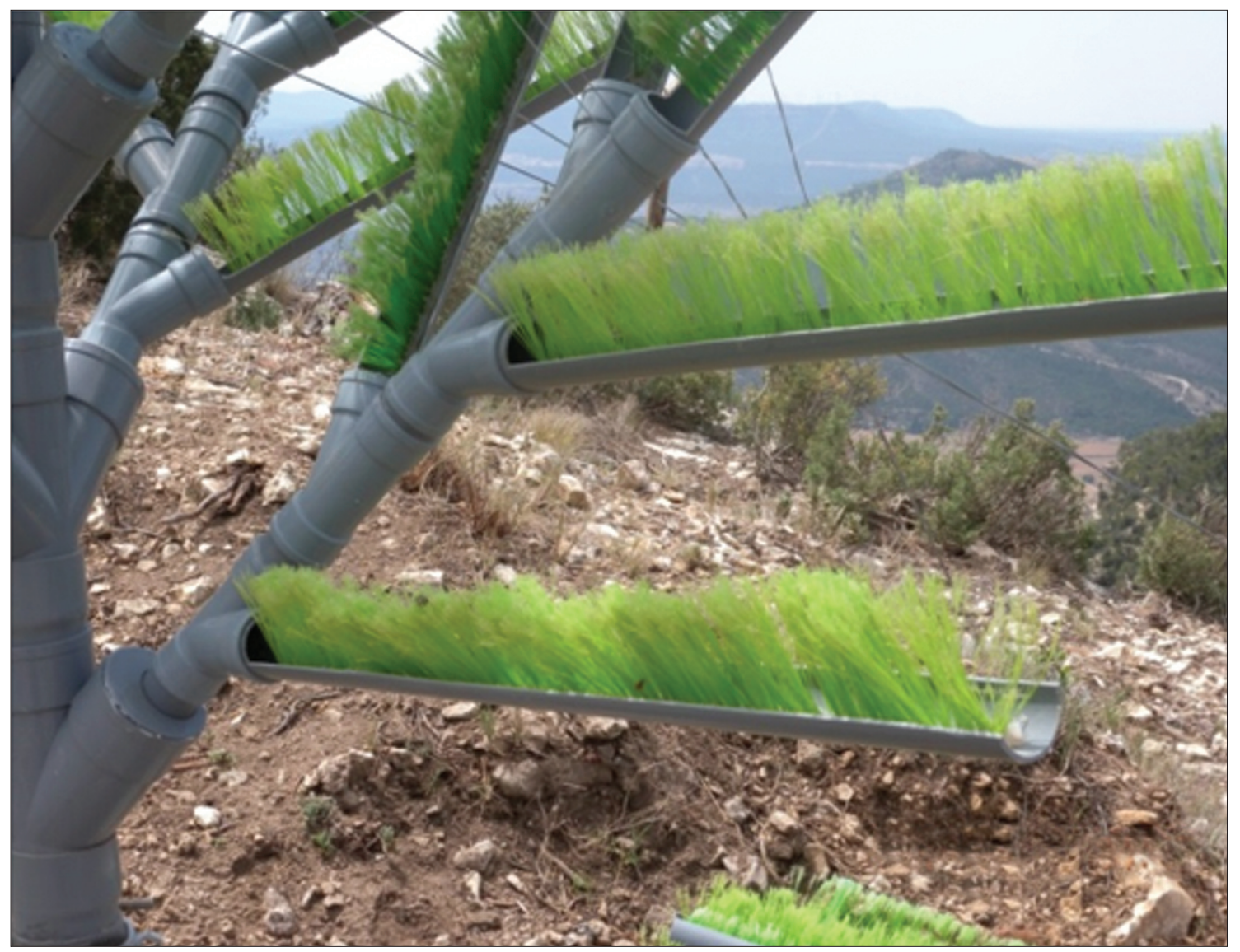

Figura 12. Detalle del método experimental de captación de agua proveniente de precipitación horizontal donde se observa el método de inserción de las ramas en el brazo y del brazo en el eje central.

8 meses, alrededor de 3501 cada día de niebla en los 8 meses que dura la serie de datos.

Una vez captada el agua se conduce por el interior del eje central mediante una tubería de PVC DN150 de alrededor de $4 \mathrm{~m}$ de longitud hasta un depósito metálico de dimensiones $1 \times 1.5 \times 0.75 \mathrm{~m}^{3}$, dicho depósito está revestido de mortero de cemento junto con unas piedras para disminuir la posible afección visual que pueda crear el depósito metálico, así como para protegerlo de posibles daños que puedan causar la fauna, y a continuación el agua se canaliza a través de una tubería hasta un bebedero realizado del mismo material que el recubrimiento del depósito metálico, según se observa en la Figura 13. Asimismo, también se dispondrá de un sistema de boya que regule la entrada del agua en el bebedero.

Como se ha comentado anteriormente se trata de un método experimental, de tal forma que sería necesario realizar una fase de pruebas situando varios árboles captadores de niebla en diferentes lugares para evaluar el potencial real tanto del método de captación como de las diferentes localizaciones, debido a que el potencial de captación que se ha comentado anteriormente no se corresponde con la realidad ya que los datos de niebla que se han tomado de referencia no se han toma- 


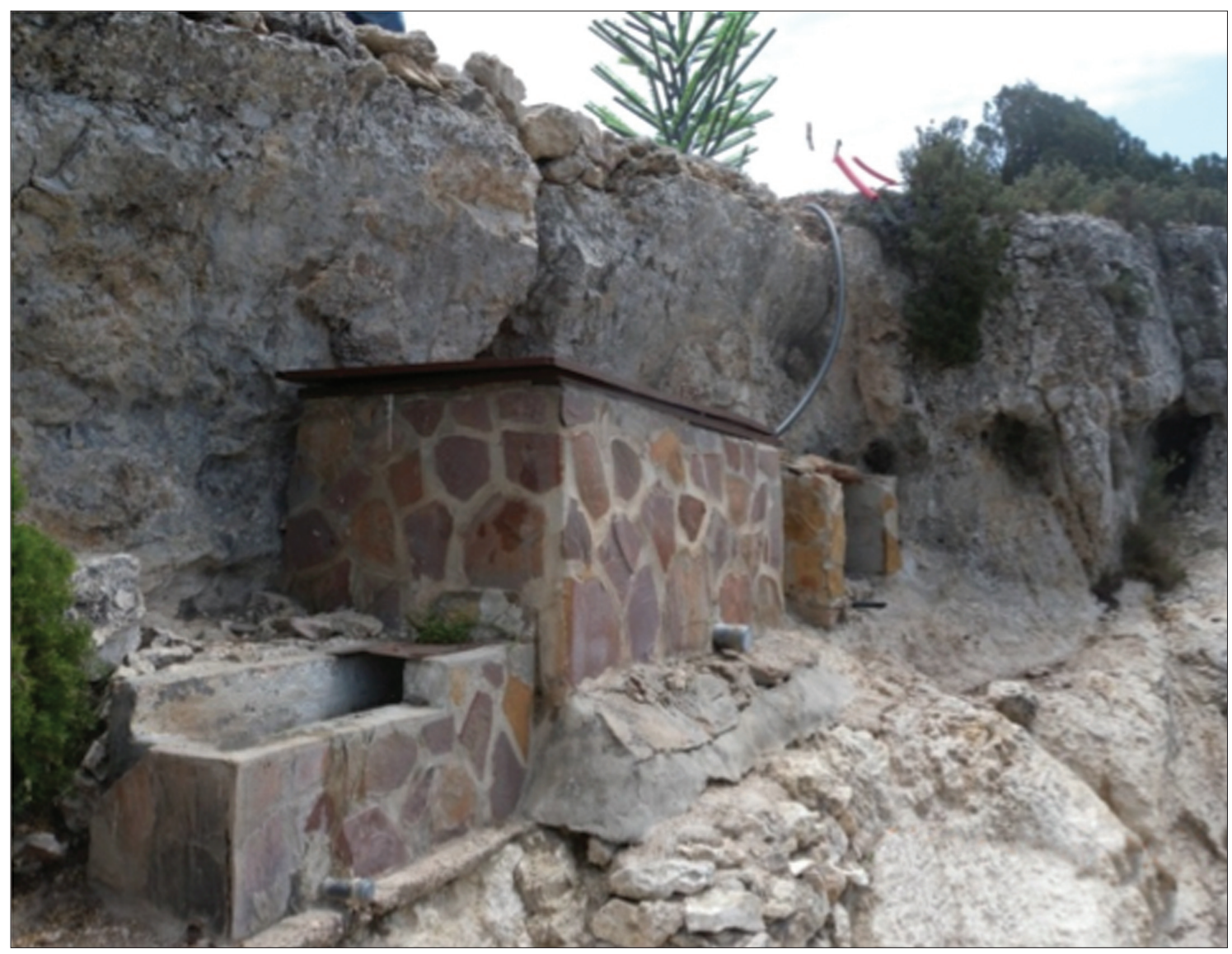

Figura 13. Imagen del método experimental de captación de agua proveniente de precipitación horizontal donde se muestra la conducción hasta un depósito de aproximadamente $1.2 \mathrm{~m} 3 \mathrm{y}$ el abrevadero.

do con el mismo captador con el que se pretende solucionar el problema de abastecimiento de la fauna, por lo tanto es imprescindible una fase de verificación de resultados.

\subsection{Otras alternativas}

Se han analizado otras alternativas, que básicamente han sido:

- El aprovechamiento de la balsa Los Calderones, actualmente fuera de servicio.

- Aprovechamiento de escorrentías recogidas en caminos rurales, como ya se está haciendo en la actualidad.

- Intensificación en el uso de los recursos procedentes de la Balsa de la Muela $\left(20 \mathrm{hm}^{3}\right)$, que ya se están aprovechando en la actualidad.

\subsection{Costes}

Los costes estimados individuales de cada una de las alternativas analizadas se resumen en la Tabla 8: 
Tabla 8. Tabla resumen del coste individual de cada alternativa

\begin{tabular}{ll}
\hline Alternativa & Coste estimado (€) \\
\hline Tractor agrícola con cuba del orden de $10 \mathrm{~m}^{3}$ & $100,000.00$ \\
\hline Charca de tierra & $155,311.02$ \\
\hline Charca de hormigón & $229,705.99$ \\
\hline Cuenca artificial de $400 \mathrm{~m}^{2}$ & $25,718.17$ \\
\hline Cubierta de entre $100-200 \mathrm{~m}^{2}$ con depósito sintético & $9,611.05$ \\
\hline Cubierta de entre $200-300 \mathrm{~m}^{2}$ con depósito sintético & $13,335.89$ \\
\hline Cubierta de entre $300-400 \mathrm{~m}^{2}$ con depósito sintético & $16,567.73$ \\
\hline Cubierta de más de $400 \mathrm{~m}^{2}$ con depósito sintético & $20,634.17$ \\
\hline Cubierta de más de $400 \mathrm{~m}^{2}$ con depósito de hormigón & $13,485.5$ \\
\hline Ud. De Árbol captador de niebla & $1,900.00$ \\
\hline
\end{tabular}

\section{Conclusiones}

La gestión cinegética tiene cuatro pilares fundamentales que se exponen a continuación, el primero es la disponibilidad de alimento, el segundo es la disponibilidad de agua, el tercero es el control de enfermedades y el cuarto es el control de depredadores. Dentro de estos cuatro bloques los dos últimos no resultan problemáticos en la actualidad en la Reserva Valenciana de Caza de la Muela de Cortes. Sin embargo la falta de alimento y la escasez de recursos hídricos constituyen una seria limitación para la gestión eficaz de la Reserva, motivo por el cual surge el presente trabajo que se ha centrado exclusivamente en la propuesta de infraestructuras hidráulicas para el abastecimiento de la fauna, dejando fuera del mismo el estudio y solución a la escasez de alimento.

Por lo tanto las conclusiones extraídas son:

1. Según nuestro criterio, no existe una solución única aplicable a toda la Reserva para resolver el problema del abastecimiento de agua debido a la heterogeneidad del territorio así como de los diferentes métodos potencialmente aplicables en cada zona, y la variabilidad pluviométrica, por ello se ha optado por diseñar, y cuantificar las distintas alternativas y una primera aproximación a su zonificación.

2. La solución exige la disponibilidad presupuestaria por parte de la Administración competente.

3. Los depósitos y charcas no son una solución en periodos de sequía extrema.

4. La solución del tractor con cuba, en combinación con una red de depósitos convenientemente distribuida, es la única alternativa que puede garantizar el abastecimiento en periodos de sequía extrema. 
5. Las charcas son una excelente solución en años de pluviometría media ya que permiten acortar notablemente las distancias y tiempos de transporte desde los puntos de captación hasta los depósitos de distribución y por lo tanto abaratar los costes derivados del transporte.

6. La alternativa de las casas puede presentar diversos inconvenientes, como la disponibilidad por parte de su propietario, y la posible rehabilitación de ellas, lo cual supone un coste añadido.

7. La alternativa de "cuenca impermeable", presenta menores problemas que la de las casas y ofrece mejores soluciones, como la libertad en la ejecución de la misma, la elección de su emplazamiento y la adaptación al terreno del conjunto superficie captante depósito de acumulación.

8. Se debe de seguir profundizando en la investigación y desarrollo de la captación de precipitaiones horizontales.

9. La adecuación y mejora de las infraestructuras existentes, sobre todo la disponibilidad de un pequeño caudal continuo desde la balsa de "la Muela", y el aprovechamiento de la balsa de "los Calderones" supondría la disposición de dos importantes puntos de agua a un coste ínfimo.

10 La alternativa de las charcas, podría ser interesante considerarla para el caso de la existencia de pequeñas fuentes, permanentes o temporales, combinada con la captación de escorrentía superficial ya que permitiría acumular un volumen importante para ser utilizado el resto del año.

\section{Bibliografía}

Anaya Garduño, M. (2011). Captación del agua de lluvia. Solución caída del cielo. México, D.F.: Mundi Prensa México.

Corell, D., Estrela, M., \& Valiente, J. (2011). Obtención de agua a partir de la niebla en el Montnegre y en otros lugares del litoral mediterráneo.

Gumbel, E. (1967). Statistics of extremes. New York (USA): Columbia University Press.

Instituto Nacional de Reforma y Desarrollo Agrario-Ministerio de Agricultura, Pesca y Alimentación. (1985). Diseño y cosntrucción de pequeños embalses. Madrid: Neografis, S.L.

Lluna, A. (2012). Alternativas para la mejora de la disponibilidad y distribución del recurso hídrico en la RVC Muela de Cortes. Valencia: Universidad Politécnica de Valencia.

Ministerio de Obras Públicas. (1967). Instrucción para el proyecto, construcción y explotación de grandes presas. Madrid: Dirección General de Obras Hidráulicas.

Témez Peláez, J. (1978). Cálculo hidrometereológico de caudales máximos en pequeñas 
cuencas naturales. Madrid: Dirección General de Carreteras (Ministerio de Obras Públicas y Urbanismo).

Unites States Departament of Agriculture (USDA). (2004). National Enginering Handbook. Part 630: Hidrology. Washington, D. C.: National Resources Conservation Service (NRCS).

VAERSA-Generalitat Valenciana . (2012). Plan de Ordenación Cinegética de la Reserva Valenciana de Caza de la Muela de Cortes. Valencia: Conselleria de Infraestructuras, Territorio y Medio Ambiente.

Vallarino Cánovas del Castillo, E. (1991). Tratado básico de presas. Madrid: Colegio de Ingenieros de Caminos, Canales y Puertos. 